\title{
Effect of Low Back Pain Risk-Stratification Strategy on Patient Outcomes and Care Processes: the MATCH Randomized Trial in Primary Care
}

\author{
Dan Cherkin, $P h D^{7}$, Benjamin Balderson, $P h D^{7}$, Rob Wellman, $M S^{7}$, Clarissa Hsu, $P h D^{1,2}$, \\ Karen J. Sherman, $P h D^{7}$, Sarah C. Evers, $M P H^{7}$, Rene Hawkes, BS ', Andrea Cook, PhD', \\ Martin D. Levine, $M D^{3}$, Diane Piekara, $P T^{7}$, Pam Rock, $P T^{7}$, Katherine Talbert Estlin, $\mathrm{MD}^{4}$, \\ Georgie Brewer ${ }^{5}$, Mark Jensen ${ }^{5}$, Anne-Marie LaPorte ${ }^{5}$, John Yeoman ${ }^{5}$, Gail Sowden, MSc 6 , \\ Jonathan C. Hill, PhD', and Nadine E. Foster, DPhil
}

${ }^{1}$ Kaiser Permanente Washington Health Research Institute, Seattle, WA, USA; ${ }^{2}$ Center for Community Health and Evaluation, Seattle, WA, USA; ${ }^{3}$ lora Health, Lynwood, WA, USA; ${ }^{4}$, Fortuna, USA; ${ }^{5}$, Seattle, WA, USA; ${ }^{6}$ Arthritis Research UK Primary Care Centre, Research Institute for Primary Care and Health Sciences, Keele University, Keele, UK.

BACKGROUND: The STarT Back strategy for categorizing and treating patients with low back pain (LBP) improved patients' function while reducing costs in England.

OBJECTIVE: This trial evaluated the effect of implementing an adaptation of this approach in a US setting.

DESIGN: The Matching Appropriate Treatments to Consumer Healthcare needs (MATCH) trial was a pragmatic cluster randomized trial with a pre-intervention baseline period. Six primary care clinics were pair randomized, three to training in the STarT Back strategy and three to serve as controls.

PARTICIPANTS: Adults receiving primary care for nonspecific LBP were invited to provide data 2 weeks after their primary care visit and follow-up data 2 and 6 months (primary endpoint) later.

INTERVENTIONS: The STarT Back risk-stratification strategy matches treatments for LBP to physical and psychosocial obstacles to recovery using patient-reported data (the STarT Back Tool) to categorize patients' risk of persistent disabling pain. Primary care clinicians in the intervention clinics attended six didactic sessions to improve their understanding LBP management and received in-person training in the use of the tool that had been incorporated into the electronic health record (EHR). Physical therapists received 5 days of intensive training. Control clinics received no training.

MAIN MEASURES: Primary outcomes were back-related physical function and pain severity. Intervention effects were estimated by comparing mean changes in patient outcomes after 2 and 6 months between intervention and control clinics. Differences in change scores by trial arm and time period were estimated using linear mixed effect models. Secondary outcomes included healthcare utilization.

KEY RESULTS: Although clinicians used the tool for about half of their patients, they did not change the

Prior Presentations Evaluation of Implementation of a Risk Stratification Strategy to Improve Primary Care for Low Back Pain. International Back and Neck Pain Research Forum, Oslo, Norway, 14 September 2017.

Received September 1, 2017

Revised January 3, 2018

Accepted April 11, 2018

Published online May 22, 2018 treatments they recommended. The intervention had no significant effect on patient outcomes or healthcare use. CONCLUSIONS: A resource-intensive intervention to support stratified care for LBP in a US healthcare setting had no effect on patient outcomes or healthcare use.

TRIAL REGISTRATION: National Clinical Trial Number NCT02286141.

KEY WORDS: low back pain; risk-stratification; STarT Back; primary care; patient outcomes.

$\begin{array}{ll}\text { Abbreviations } \\ \text { CAM } & \text { Complementary and alternative medicine } \\ \text { CBT } & \text { Cognitive behavioral therapy } \\ \text { DVD } & \text { Digital video disc } \\ \text { CATI } & \text { Computer-assisted telephone interview } \\ \text { CME } & \text { Continuing medical education } \\ \text { EHR } & \text { Electronic health record } \\ \text { GAD } & \text { Generalized anxiety disorder } \\ \text { GH } & \text { Group Health } \\ \text { GLMM } & \text { Generalized linear mixed models } \\ \text { LMM } & \text { Linear mixed models } \\ \text { MATCH } & \text { Matching Appropriate Treatments to Consumer Health- } \\ & \text { care needs } \\ \text { NIH } & \text { National Institutes of Health } \\ \text { PC } & \text { Primary care } \\ \text { PCORI } & \text { Patient-Centered Outcomes } \\ \text { PCP } & \text { Primary care provider } \\ \text { PGIC } & \text { Patient Global Impression of Change } \\ \text { PHQ } & \text { Patient health questionnaire } \\ \text { PSEQ } & \text { Pain self-efficacy questionnaire } \\ \text { PT } & \text { Physical therapy/physical therapist } \\ \text { RMDQ } & \text { Roland-Morris Disability Questionnaire } \\ \text { RS } & \text { Risk stratification } \\ \text { STarT } & \text { Subgroups for Targeted Treatment” Risk Stratification } \\ \text { TSK } & \text { Tampa Scale of Kinesiophobia } \\ \text { UC } & \text { Usual care } \\ \text { WPAI } & \text { Work Productivity and Activity Impairment } \\ & \end{array}$

$\mathrm{J}$ Gen Intern Med 33(8):1324-36

DOI: $10.1007 / \mathrm{s} 11606-018-4468-9$

(c) Society of General Internal Medicine 2018 


\section{INTRODUCTION}

Despite increasing US expenditures for low back pain (LBP), patient outcomes have deteriorated. ${ }^{1}$ The current epidemic of opioid addiction and deaths illustrates the urgency of finding safer and more effective approaches for chronic pain. ${ }^{2,3}$ The traditional view of LBP as a largely biomedical problem ${ }^{4}$ is being supplanted by the biopsychosocial model that acknowledges that while pain usually has an underlying biological basis, psychosocial factors (e.g., pain beliefs/cognitions, distress, coping behaviors, and social factors) also significantly influence the experience and impact of pain. ${ }^{5,6}$ This broader conceptualization provides a clear rationale for incorporating cognitive behavioral principles into the management of distressed and disabled patients with chronic LBP to minimize pain-related disability.

A promising strategy for categorizing and treating patients considering both their physical and psychosocial characteristics, the STarT Back approach, was developed and evaluated in England. ${ }^{7}$ This approach improved patients' physical function and satisfaction with care while reducing costs. ${ }^{8-11}$ This strategy uses patient responses to a nine-item "STarT Back tool" questionnaire to allocate patients to a low-, medium-, or high-risk subgroup according to their risk of persistent disabling back pain. Patients in each subgroup are then recommended evidence-based treatments matched to their prognostic profile. ${ }^{7,12,13}$ Patients found to have at least four out of the five "psychosocial" risk factors (high pain bothersomeness, fear, worry, catastrophizing, depression) are "high risk" and those with relatively few (0-3) physical or psychosocial risk factors are "low risk." The remaining patients with significant pain and/or activity limitations but fewer psychosocial risk factors are "medium risk." This tool has been validated with primary care adults with non-specific LBP. ${ }^{12}$ The success of this strategy in England. has generated great interest in developed countries, providing new hope that meaningful improvements in primary care for LBP are within reach. ${ }^{14-19}$

Prior to widespread implementation, it would be valuable to know if the STarT Back strategy can be successfully translated to other settings. To determine if the STarT Back risk stratification strategy would succeed in the USA, we conducted the Matching Appropriate Treatments to Consumer Healthcare needs (MATCH) cluster randomized trial. This trial evaluates the effects of incorporating the STarT Back strategy into primary care practices within an integrated healthcare system. The goal was to give primary care providers (PCPs) and physical therapists the knowledge, tools, and confidence they needed to provide their patients with a broader understanding of their LBP, reassurance about their likely prognosis, and treatment options that matched the patients' prognostic profile. We hypothesized this intervention would improve patient outcomes by promoting increased use of matched treatment options for patients in each subgroup, as determined by the STarT Back tool. We believe this is the first randomized and controlled evaluation of the STarT Back approach in the USA.

\section{METHODS}

\section{Design and Setting}

The trial design has been reported in detail. ${ }^{20}$ In brief, MATCH was a pragmatic, cluster randomized trial with two parallel $\mathrm{arms}^{21}$ each with a baseline data collection period. Six primary care clinics were randomized in 1:1 ratio to intervention or control (Fig. 1). PCPs were either MDs (84\%) or physician assistant/nurse practitioners (16\%); $85 \%$ had practiced over 5 years; and $62 \%$ were female. Data were collected from patients and electronic health records (EHR). The trial was conducted in Group Health (GH), an integrated healthcare delivery system in Washington State serving over 600,000 members GH is now called kaiser permanente washington.

Clinical leaders at GH partnered with the research team to evaluate the effect of stratified care in its primary care clinics. The intervention was incorporated into a mandatory care improvement activity, fully supported by clinical and administrative leadership.

\section{Participating Clinics (Clusters) and Patients}

One clinic from each of three pairs of large primary care clinics (with onsite physical therapy) near Seattle matched on geographic and socioeconomic characteristics, were pair randomized to the intervention or control. Control clinics received no intervention. Pre-intervention levels of patient outcomes were measured in all six clinics. The intervention was then implemented in intervention clinics over 6 months, after which patient outcomes were again assessed in both intervention and control clinics.

During the trial, all patients $18+$ years of age identified in the EHR as having received a primary diagnosis consistent with non-specific LBP (e.g., lumbago, back pain not otherwise specified) were eligible to participate. To maintain broad applicability, we only excluded patients with specific causes of pain (e.g., pregnancy, disc herniation, vertebral fracture, spinal stenosis) or with job injuries, which were seen in the Occupational Medicine clinic.

\section{Randomization and Blinding}

Prior to the intervention, the trial biostatistician randomly assigned one clinic in each of the three geographic and sociodemographic matched pairs of clinics to the intervention by computer-generated random number. All eligible patients seen in the intervention clinics were considered to have received the intervention. Researchers did not inform patients that their clinics were participating in an intervention. Interviewers were blinded to patients' clinics.

\section{The Intervention}

The intervention was implemented in the 3 intervention clinics from April-September 2014. Key components of the intervention were incorporating the original version of the STarT Back 


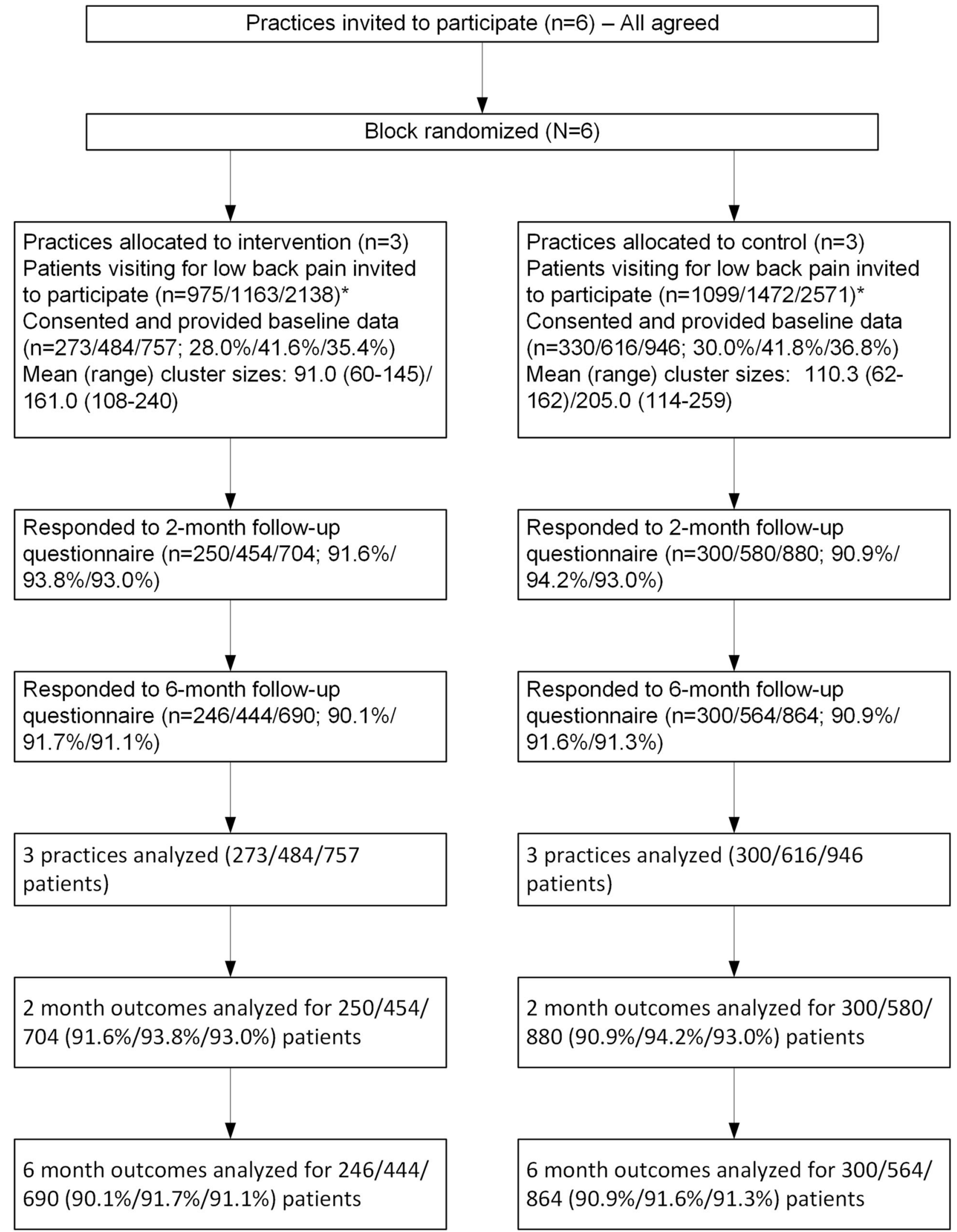

* Data are presented for the pre-intervention period/post-intervention period/total of the two periods

Fig. 1 Flow of patients through trial. 
tool $^{8}$ into the EHR, identifying recommended treatment options available from $\mathrm{GH}$ for patients in each risk subgroup, and training the primary care teams and physical therapists (Appendix 1 online). ${ }^{20}$

\section{Outcomes}

Patient outcome data were collected by telephone interviewers during the pre-intervention (November 2013-April 2014) and post-intervention (December 2014-August 2016) periods. Interviews occurred 2 weeks (range, 1 to 3 weeks) after the LBP visit (baseline) and again 2 and 6 months later. Primary outcomes were LBP-related physical function in the previous week (measured with the modified Roland-Morris Disability Questionnaire (RMDQ) ${ }^{22}$ ) and LBP severity during the previous week (measured on a 0 -to-10 scale where 0 represents "no pain" and 10 "pain as bad as it could be"). ${ }^{23}$ Secondary outcomes included patient outcomes (depression, anxiety, fear of movement, global improvement, self-efficacy, satisfaction, and work productivity and activity impairment ${ }^{20}$ ) and actual healthcare utilization from the automated EHR (e.g., lumbar imaging, physical therapy, complementary and alternative medical therapies, cognitive behavioral therapy, opioids, spinal injections, and spine surgeon consultations). Because the intervention targeted PCPs, we could not identify adverse effects.

\section{Data Collection}

The pre-intervention period was devoted to measuring changes in patient outcomes 2 and 6 months after LBP visits in the intervention and control clinics. Because collecting baseline data during the visit was not feasible, we mailed patients letters shortly after the visits explaining GH was conducting a study to improve LBP care and that we would call to invite their participation. Patients not wishing to be contacted were provided a phone number to opt out. Research specialists called patients between 1 and 3 weeks after their visit to explain the study, answer questions, confirm eligibility and obtain verbal informed consent to complete a baseline and two follow-up interviews. Patients were paid \$20 for each questionnaire. Trained interviewers used computer-assisted telephone interviewing to minimize errors and missing data. Similar methods were used to collect post-intervention data. Because we did not meet our recruitment goal during the pre-intervention period, we increased post-intervention recruitment to maintain overall statistical power (see "Sample Size" section). We also improved the recruitment letter, increased staffing, and lengthened the recruitment period. The mean interval between visit date and baseline data collection was $12.7(\mathrm{SD}=7.1)$ days.

\section{Sample Size}

A priori sample size calculations were performed targeting $80 \%$ power to detect a 1.5-point difference in 6-month LBP-related change in patient function (RMDQ) pre- and post-intervention between control and intervention clinics ( 0 point difference in the low risk subgroups and 2.5 points difference in mediumand high-risk subgroups) and 0.9 points on LBP pain severity score ( 0 point difference in low risk subgroups and 1.5 points difference in medium- and high-risk subgroups). We planned for a sample size of 1760 participants balanced equally between the pre- and post-intervention periods and the control and intervention clinics allowing for a loss to follow-up rate of $20 \% .^{20}$ Because we recruited only 603 participants (goal was 880) during the pre-intervention period, we determined that we would need a sample size of 1334 during the post-intervention period to maintain $80 \%$ power. The final numbers recruited were 603 participants in the pre-intervention period (546 with complete follow-up) and 1098 in the post-intervention period (1008 with complete follow-up). Our post hoc calculation of power based on the observed data (accounting for imbalance between intervention arms) found we had $80 \%$ power to detect a difference between trial arms of 1.5 points on the change in RMDQ score before and after the intervention. We assumed no correlation of outcomes within provider or clinic.

\section{Statistical Methods}

We first estimated the change in mean score by clinic assignment between the pre- and post-intervention periods. We then compared these differences to estimate the change attributable to the intervention (i.e., we made inferences on the interaction between clinic assignment and intervention period). We used a linear mixed effects model with random effects ${ }^{24}$ for patient participants (repeated outcome measurements on participants at 2 and 6 months post-LBP visit) and clinic (randomization at clinic level) to account for correlation within individuals and clinics. The primary analysis time-point was 6 months following the LBP visit. To account for potential confounding variables, we adjusted for participant-level baseline covariates shown to be associated with LBP physical function and pain intensity, as well as variables that were imbalanced at baseline at the patient level between intervention and control arms: sex, age, education, race, employment, function (RMDQ), and pain intensity. Risk-subgroup-specific estimates and secondary outcomes were calculated using an identical framework to that described above with one exception. For binary secondary outcomes, we used generalized linear mixed models $(\mathrm{GLMM})^{25}$ with logit and/or log link functions to estimate odds ratios and/or relative risks instead of mean change scores. We assumed the standard alpha level of 0.05 for a two-sided test.

We used the same analytic approach with EHR data to evaluate the effect of the intervention on healthcare utilization for LBP. We examined if the use of recommended treatments for patients at medium and high risk of persistent disabling pain increased and the use of treatments generally not recommended for non-specific LBP decreased. The primary analyses included all eligible patients (not just those providing patient data). We also analyzed data for the subset of patients who participated in the telephone questionnaires. Comparison 
of the data from these two populations allowed us to determine the representativeness of participants. We also examined the frequency with which STarT Back risk scores were recorded in the EHR. See ref. ${ }^{20}$ for more detail.

\section{RESULTS}

\section{Patient Recruitment and Follow-up}

Figure 1 presents the flow diagram showing the six clinics in this cluster RCT and the flow of trial participants. Because we included a pre-intervention "baseline" period, we present flow data for both the pre- and post-intervention periods as well as for the total. A total of 2138 LBP patients visited the intervention clinics and 2571 the control clinics. Characteristics of intervention and control patients were similar both pre- and post-intervention. Overall, $36 \%$ of eligible patients provided baseline data on the telephone. Participating patients were slightly older than non-participants (mean ages of 57.1 and 54.8 , respectively) and more likely to be white (83.0 and $77.0 \%$, respectively) but did not differ by gender. Follow-up rates were $93 \%$ at 2 months and $91 \%$ at 6 months. Participation and follow-up rates were similar in the intervention and control clinics.

\section{Patient Characteristics}

Reflecting the GH membership, participants had relatively high levels of education and income and were primarily white and non-Hispanic (Table 1). About half the participants were over 60 years old. Participants had moderately high levels of functional disability and pain severity, $56 \%$ had episodes lasting less than 3 months, $48 \%$ reported leg pain, and about $30 \%$ were using opioids for their pain. Data from the STarT Back tool showed that $41 \%$ were categorized at low risk, $37 \%$ at medium risk, and $22 \%$ at high risk of persistent disabling pain. The STarT Back tool successfully predicted the prognoses of the three risk groups (i.e., the high-risk group had the worst outcomes and the low-risk group had the best outcomes). ${ }^{26}$ Participants' characteristics were similar in the intervention and control arms, showing no evidence of selection bias.

\section{Effect of the Intervention}

Patient Outcomes. At 6 months, there were no statistically significant differences between participants in the intervention and control arms for either primary patient outcome overall or within risk subgroup (Table 2) or for secondary patient outcomes (Table 3). The absolute magnitudes of the between group differences were small and for the primary outcome measures slightly favored the control group. Similar results were found at 2 months (Appendix 2 online).

Healthcare Utilization. STarT Back tool data were available for about $50 \%$ of LBP visits during the 6-month intervention
Table 1 Baseline characteristics of study participants in the control $(n=3)$ and intervention $(n=3)$ clinics

\begin{tabular}{|c|c|c|}
\hline Characteristic & $\begin{array}{l}\text { Control } \\
(n=945)\end{array}$ & $\begin{array}{l}\text { Intervention } \\
(N=756)\end{array}$ \\
\hline Sex (female, No. (\%)) & $512(54.2)$ & $441(58.3)$ \\
\hline Age (mean (SD), year) & $55(17.3)$ & $58(18.4)$ \\
\hline 18-39 (No. (\%)) & $215(22.7)$ & $160(21.1)$ \\
\hline 40-59 (No. $(\%))$ & $310(32.8)$ & $204(26.9)$ \\
\hline $60+($ No. $(\%))$ & $420(44.4)$ & $392(51.8)$ \\
\hline \multicolumn{3}{|l|}{ Education } \\
\hline High school or less (No. (\%)) & $143(15.1)$ & $105(13.9)$ \\
\hline Some college $($ No. $(\%))$ & $303(32.1)$ & $231(30.6)$ \\
\hline College/post-graduate (No. (\%)) & $498(52.8)$ & $418(55.4)$ \\
\hline \multicolumn{3}{|l|}{ Income* } \\
\hline$<\$ 35 \mathrm{~K}($ No. $(\%))$ & $161(18.3)$ & $141(20.2)$ \\
\hline$\$ 45-55 \mathrm{~K}($ No. $(\%))$ & $203(23.1)$ & $186(26.6)$ \\
\hline$\$ 55-85 \mathrm{~K}($ No. $(\%))$ & $216(24.6)$ & $149(21.3)$ \\
\hline$\$ 85 \mathrm{~K}+($ No. $(\%))$ & $298(33.9)$ & $223(31.9)$ \\
\hline Employed (No. (\%) & 557 (58.9) & $417(55.2)$ \\
\hline White (No. $(\%))^{*}$ & 743 (79.9) & $582(78.0)$ \\
\hline Hispanic (No. (\%))* & $53(5.8)$ & $40(5.5)$ \\
\hline $\begin{array}{l}\text { Back-related function (RMDQ; } \\
0-23 \text { scales) mean (SD) }\end{array}$ & $11.8(6.3)$ & $11.8(6.1)$ \\
\hline $\begin{array}{l}\text { Back pain severity }(0-10 \text { scales; } \\
\text { mean (SD) })^{t}\end{array}$ & $5.4(2.5)$ & $5.5(2.5)$ \\
\hline \multicolumn{3}{|l|}{ STarT back risk group } \\
\hline Low (No. $(\%)$ ) & $392(41.5)$ & $305(40.3)$ \\
\hline Medium (No. (\%)) & $348(36.8)$ & $286(37.8)$ \\
\hline High (No. (\%)) & $205(21.7)$ & $165(21.8)$ \\
\hline \multicolumn{3}{|l|}{$\begin{array}{l}\text { Duration of current episode } \\
\text { of LBP }\end{array}$} \\
\hline$<3$ months $(\%)$ & $186(56,4)$ & $153(56.0)$ \\
\hline $3-12$ months (\%) & $58(17.6)$ & $47(17.2)$ \\
\hline 12 months (\%) & $86(26.0)$ & $73(26.7)$ \\
\hline $\begin{array}{l}\text { Hrs. of work missed past week } \\
\text { due to LBP (mean (SD)) }\end{array}$ & $4.4(10.4)$ & $3.6(8.9)$ \\
\hline $\begin{array}{l}\text { Effect of LBP on work in past } \\
\text { week }(0-10 ; \text { mean }(S D))\end{array}$ & $3.0(2.9)$ & $3.0(2.7)$ \\
\hline Leg pain in leg (No. $(\%)$ ) & $457(48.4)$ & $360(47.7)$ \\
\hline Anxiety (GAD-7; mean (SD)) & $4.2(4.6)$ & $4.4(4.6)$ \\
\hline Depression (PHQ-8; mean (SD)) & $6.1(5.4)$ & $6.2(5.3)$ \\
\hline Self-efficacy (PSEQ; mean (SD)) & $44.4(13.3)$ & $45.0(12.7)$ \\
\hline Fear of movement (TKS; mean (SD)) & $39.7(10.1)$ & $39.4(10.3)$ \\
\hline $\begin{array}{l}\text { Used medications for back pain } \\
\text { in past week (No. }(\%))\end{array}$ & $735(77.8)$ & $570(75.5)$ \\
\hline $\begin{array}{l}\text { Used narcotics for back pain } \\
\text { in past week (No. }(\%))\end{array}$ & $104(31.5)$ & $76(27.8)$ \\
\hline
\end{tabular}

Abbreviations: $L B P$, low back pain; GAD, generalized anxiety disorder; $P H Q$, patient health questionnaire; PSEC, patient self-efficacy questionnaire; TSK, Tampa Scale for Kinesiophobia; RMDQ, Roland-Morris Disability Questionnaire

*Missing data: income and pain duration (7\%), race and Hispanic $(3 \%)$, and hours of work missed (1\%). All other variables had $<1 \%$ missing

${ }^{t}$ Higher scores indicate greater dysfunction

${ }^{*}$ Higher scores indicate greater pain severity

${ }^{\xi}$ Pain duration was measured only during the pre-implementation period, resulting in smaller sample sizes than for the other measures

period, decreasing to about $40 \%$ over the ensuing 20 months. Among the 32 PCPs in the intervention clinics who saw at least ten patients with LBP during both the intervention period and post-intervention period, the median percentage of visits with a STarT Back tool score in the EHR was 47\% (range 23$71 \%$ ) during the intervention period and $42 \%$ (range $8-71 \%$ ) during the post-intervention period. Thus, the tool continued to be used for patients of all PCPs, at least occasionally, long after the intervention period ended.

Despite PCP or nursing staff entry of the STarT Back tool data for almost half of the visits for LBP, knowledge of the 
Table 2 Primary outcomes main analysis and by risk subgroup at 6-month follow-up

\begin{tabular}{|c|c|c|c|c|c|c|c|c|}
\hline & \multicolumn{3}{|c|}{ Pre-intervention period } & \multicolumn{3}{|c|}{ Post-intervention period } & \multirow[t]{2}{*}{ Difference } & \multirow[t]{2}{*}{$95 \% \mathrm{CI}$} \\
\hline & $N$ & Change & $95 \% \mathrm{CI}$ & $N$ & Change & $95 \% \mathrm{CI}$ & & \\
\hline \multicolumn{9}{|l|}{ Function (RMDQ) } \\
\hline Control clinics & 297 & -3.33 & \multicolumn{6}{|c|}{ Main analysis } \\
\hline Intervention clinics & 245 & -3.98 & $(-4.86,-3.09)$ & 428 & -4.03 & $(-4.81,-3.25)$ & -0.05 & $(-0.83,0.73)$ \\
\hline $\begin{array}{l}\text { Difference } \\
P \text { value }\end{array}$ & & -0.64 & $(-1.86,0.58)$ & & -0.14 & $(-1.22,0.94)$ & 0.50 & $\begin{array}{r}(-0.55,1.55) \\
0.349\end{array}$ \\
\hline \multicolumn{9}{|l|}{ Subgroup, low risk } \\
\hline Control clinics & 122 & -5.18 & $(-5.98,-4.39)$ & 234 & -5.75 & $(-6.37,-5.12)$ & -0.56 & $(-1.42,0.29)$ \\
\hline Intervention clinics & 109 & -5.60 & $(-6.41,-4.78)$ & 170 & -5.60 & $(-6.29,-4.91)$ & -0.01 & $(-0.94,0.93)$ \\
\hline $\begin{array}{l}\text { Difference } \\
P \text { value }\end{array}$ & & -0.41 & $(-1.48,0.65)$ & & 0.14 & $(-0.70,0.98)$ & 0.56 & $\begin{array}{r}(-0.71,1.82) \\
0.389\end{array}$ \\
\hline \multicolumn{9}{|c|}{ Subgroup, moderate risk } \\
\hline Control clinics & 108 & -3.54 & $(-5.11,-1.97)$ & 203 & -3.90 & $(-5.30,-2.50)$ & -0.36 & $(-1.61,0.89)$ \\
\hline Intervention clinics & 88 & -3.34 & $(-4.98,-1.70)$ & 165 & -3.77 & $(-5.21,-2.32)$ & -0.43 & $(-1.82,0.96)$ \\
\hline $\begin{array}{l}\text { Difference } \\
P \text { value }\end{array}$ & & 0.20 & $(-2.06,2.46)$ & & 0.13 & $(-1.86,2.13)$ & -0.06 & $\begin{array}{r}(-1.94,1.81) \\
0.946\end{array}$ \\
\hline \multicolumn{9}{|l|}{ Subgroup, high risk } \\
\hline Control clinics & 67 & -1.42 & $(-3.33,0.49)$ & 109 & -2.49 & $(-4.15,-0.82)$ & -1.07 & $(-2.92,0.79)$ \\
\hline Intervention clinics & 48 & -3.78 & $(-5.82,-1.74)$ & 93 & -3.09 & $(-4.77,-1.41)$ & 0.69 & $(-1.46,2.84)$ \\
\hline $\begin{array}{l}\text { Difference } \\
P \text { value }\end{array}$ & & -2.36 & $(-4.77,0.05)$ & & -0.60 & $(-2.48,1.27)$ & 1.76 & $\begin{array}{r}(-1.10,4.62) \\
0.229\end{array}$ \\
\hline \multicolumn{9}{|l|}{ Pain intensity. } \\
\hline \multicolumn{9}{|l|}{ Main analysis } \\
\hline Control clinics & 297 & -1.64 & $(-1.94,-1.34)$ & 534 & -1.96 & $(-2.20,-1.72)$ & -0.32 & $(-0.66,0.01)$ \\
\hline Intervention clinics & 245 & -1.81 & $(-2.13,-1.48)$ & 415 & -2.00 & $(-2.26,-1.74)$ & -0.19 & $(-0.56,0.18)$ \\
\hline Difference & & -0.17 & $(-0.61,0.27)$ & & -0.039 & $(-0.39,0.31)$ & 0.13 & $(-0.37,0.63)$ \\
\hline$P$ value & & & & & & & & 0.61 \\
\hline \multicolumn{9}{|l|}{ Subgroup, low risk } \\
\hline Control clinics & 122 & -2.13 & $(-2.54,-1.72)$ & 228 & -2.25 & $(-2.56,-1.94)$ & -0.12 & $(-0.59,0.35)$ \\
\hline Intervention clinics & 109 & -2.27 & $(-2.69,-1.85)$ & 168 & -2.42 & $(-2.78,-2.07)$ & -0.15 & $(-0.66,0.36)$ \\
\hline Difference & & -0.14 & $(-0.69,0.41)$ & & -0.173 & $(-0.59,0.25)$ & -0.03 & $(-0.72,0.66)$ \\
\hline \multirow{2}{*}{\multicolumn{9}{|c|}{ Subgroup, moderate risk }} \\
\hline & & & & & & & & \\
\hline $\begin{array}{l}\text { Control clinics } \\
\text { Intervention clinics }\end{array}$ & 108 & -1.59 & $(-2.23,-0.95)$ & 200 & -1.93 & $(-2.48,-1.38)$ & -0.34 & $(-0.91,0.23)$ \\
\hline $\begin{array}{l}\text { Intervention clinics } \\
\text { Difference }\end{array}$ & 88 & -1.47 & $(-2.14,-0.79)$ & 157 & -1.79 & $(-2.37,-1.21)$ & -0.33 & $(-0.97,0.32)$ \\
\hline $\begin{array}{l}\text { Difference } \\
P \text { value }\end{array}$ & & 0.13 & $(-0.80,1.05)$ & & 0.14 & $(-0.65,0.93)$ & 0.02 & $(-0.85,0.88)$ \\
\hline \multicolumn{9}{|l|}{ Subgroup, high risk } \\
\hline Control clinics & 67 & -0.74 & $(-1.55,0.07)$ & 106 & -1.54 & $(-2.24,-0.84)$ & -0.8 & $(-1.62,0.03)$ \\
\hline Intervention clinics & 48 & -1.6 & $(-2.47,-0.73)$ & 90 & -1.54 & $(-2.25,-0.82)$ & 0.06 & $(-0.89,1.01)$ \\
\hline Difference & & -0.86 & $(-1.88,0.17)$ & & 0.002 & $(-0.79,0.79)$ & 0.86 & $(-0.41,2.12)$ \\
\hline$P$ value & & & & & & & & 0.183 \\
\hline
\end{tabular}

patients' risk subgroup did not affect the type or frequency of healthcare provided (Table 4). Specifically, there was no evidence that the intervention strategies used in the MATCH trial increased the use of treatments recommended for mediumand high-risk patients (e.g., physical therapy, complementary and alternative medicine, or cognitive behavioral therapy), or decreased the use of non-recommended tests or treatments (i.e., imaging, opioid medications, spine injections, surgical referrals) for LBP patients at any risk level. There was no evidence of any changes in the tests or treatments recommended by clinicians in the intervention clinics for any of the patient risk subgroups (Table 5). A pre-specified secondary analysis restricted to patients providing telephone outcome data showed similar results.

\section{DISCUSSION}

The MATCH trial is the first major evaluation of implementation of an adaption of the STarT Back risk stratification strategy in the
USA. Although the intervention resulted in use of the STarT Back tool for approximately half of patient visits for LBP, it did not change PCP treatment decisions. Another recent cluster randomized controlled trial evaluated use of a multifaceted strategy (including embedding the STarT Back Tool in the EHR) to implement LBP guidelines into Danish general practices. ${ }^{27}$ That trial found lower secondary care referral rates in the intervention clinics $(5.0 \%)$ than in the control clinics $(10.5 \%)$, but no improvement in patient outcomes.

There are many reasons complex interventions such as the one evaluated in this trial could fail to improve patient outcomes, including unacceptability to clinicians, inadequate leadership and system support, ineffective implementation, and inadequate potency. Although a comprehensive evaluation of the implementation process found high levels of clinician engagement and system support (submitted), there were limitations in our intervention that could explain why PCP behavior did not change, most notably: (1) we did not conduct feedback audits to encourage clinician adherence to matching treatments to patient subgroups and (2) compared with English studies, ${ }^{8,9}$ 


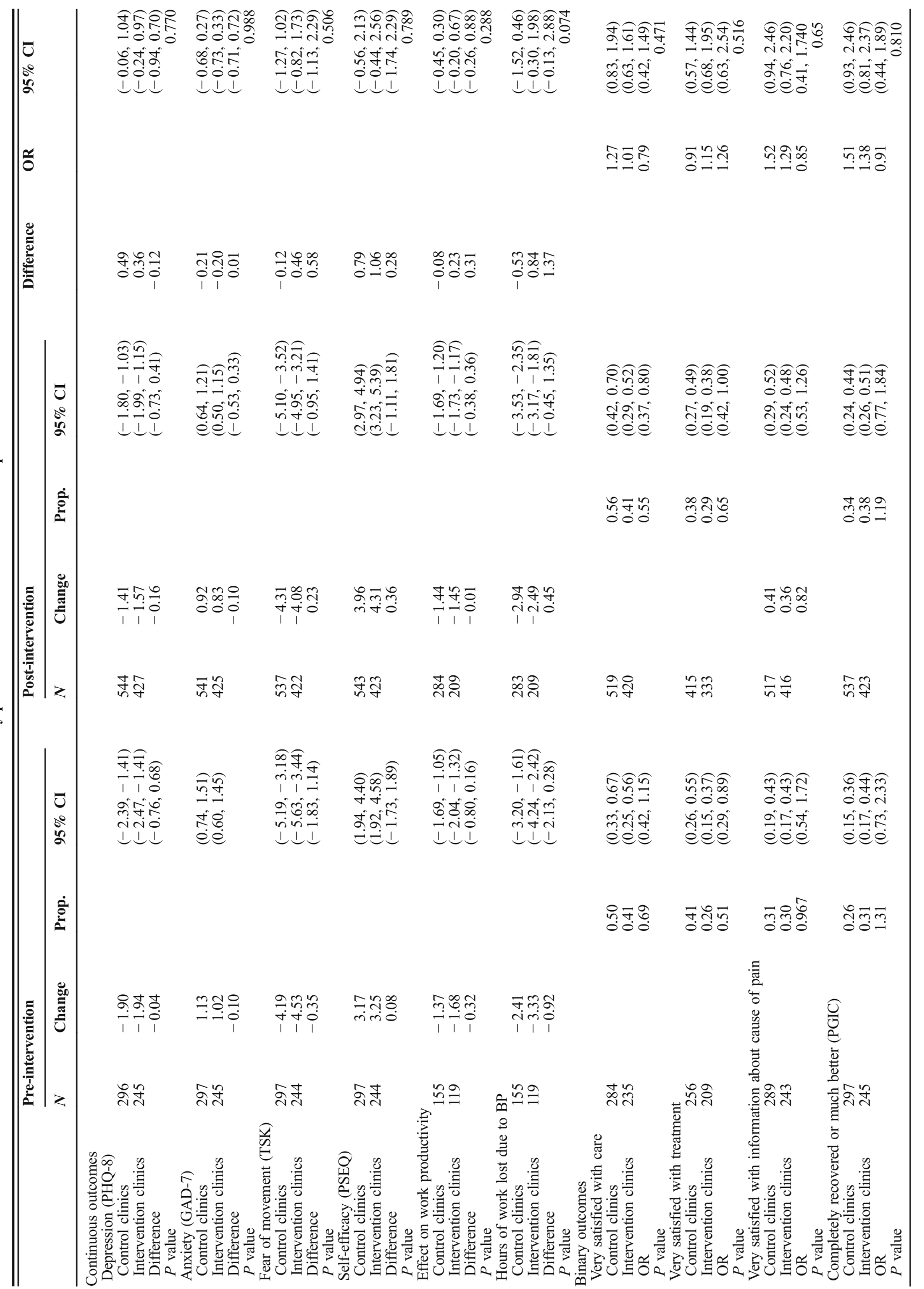


Table 4 Pre-post proportion and odds ratio (OR) for selected health services for low back pain between the control and intervention arms during the 6 months after visit

\begin{tabular}{|c|c|c|c|c|c|c|c|c|}
\hline & \multicolumn{3}{|c|}{ Pre-intervention period } & \multicolumn{3}{|c|}{ Post-intervention period } & \multirow[t]{2}{*}{ OR } & \multirow[t]{2}{*}{$95 \% \mathrm{CI}$} \\
\hline & $N$ & Prop & $95 \% \mathrm{CI}$ & $N$ & Prop & $95 \% \mathrm{CI}$ & & \\
\hline \multicolumn{9}{|c|}{ Lumbar spine imaging* } \\
\hline Control clinics & 1061 & 0.14 & $(0.07,0.22)$ & 1473 & 0.18 & $(0.09,0.27)$ & 1.34 & $(1.08,1.67)$ \\
\hline Intervention clinics & 943 & 0.16 & $(0.08,0.25)$ & 1163 & 0.22 & $(0.11,0.34)$ & 1.46 & $(1.17,1.84)$ \\
\hline $\begin{array}{l}\mathrm{OR} \\
P \text { value }\end{array}$ & & 1.20 & $(0.57,2.54)$ & & 1.31 & $(0.63,2.73)$ & 1.09 & $(0.80,1.50)$ \\
\hline \multicolumn{9}{|c|}{ Additional primary care visits } \\
\hline Control clinics & 1061 & 0.15 & $(0.12,0.17)$ & 1473 & 0.24 & $(0.21,0.27)$ & 1.86 & $(1.51,2.29)$ \\
\hline Intervention clinics & 943 & 0.12 & $(0.09,0.14)$ & 1163 & 0.24 & $(0.21,0.28)$ & 2.43 & $(1.91,3.10)$ \\
\hline $\mathrm{OR}$ & & 0.77 & $(0.59,1.01)$ & & 1.01 & $(0.82,1.23)$ & 1.31 & $(0.95,1.79)$ \\
\hline$P$ value & & & & & & & & 0.095 \\
\hline \multicolumn{9}{|c|}{ Emergency department visits } \\
\hline Control clinics & 1061 & 0.05 & $(0.04,0.06)$ & 1473 & 0.04 & $(0.03,0.05)$ & 0.77 & $(0.53,1.11)$ \\
\hline Intervention clinics & 943 & 0.04 & $(0.03,0.06)$ & 1163 & 0.03 & $(0.02,0.04)$ & 0.75 & $(0.48,1.18)$ \\
\hline OR & & 0.81 & $(0.54,1.23)$ & & 0.80 & $(0.53,1.21)$ & 0.98 & $(0.55,1.76)$ \\
\hline$P$ value & & & & & & & & 0.959 \\
\hline \multicolumn{9}{|l|}{ Narcotic analgesics } \\
\hline Control clinics & 1061 & 0.39 & $(0.30,0.48)$ & 1473 & 0.45 & $(0.35,0.55)$ & 1.28 & $(1.07,1.53)$ \\
\hline Intervention clinics & 943 & 0.37 & $(0.28,0.45)$ & 1163 & 0.41 & $(0.32,0.51)$ & 1.23 & $(1.01,1.49)$ \\
\hline OR & & 0.91 & $(0.65,1.27)$ & & 0.87 & $(0.64,1.20)$ & 0.96 & $(0.74,1.25)$ \\
\hline$P$ value & & & & & & & & 0.757 \\
\hline \multicolumn{9}{|l|}{ Physical therapy visits } \\
\hline Control clinics & 1061 & 0.21 & $(0.15,0.28)$ & 1473 & 0.22 & $(0.15,0.29)$ & 1.04 & $(0.86,1.26)$ \\
\hline Intervention clinics & 943 & 0.24 & $(0.16,0.31)$ & 1163 & 0.26 & $(0.18,0.34)$ & 1.13 & $(0.93,1.38)$ \\
\hline OR & & 1.13 & $(0.73,1.76)$ & & 1.23 & $(0.81,1.89)$ & 1.09 & $(0.83,1.43)$ \\
\hline$P$ value & & & & & & & & 0.546 \\
\hline \multicolumn{9}{|l|}{ CAM Visits } \\
\hline Control clinics & 1061 & 0.14 & $(0.12,0.17)$ & 1473 & 0.14 & $(0.12,0.16)$ & 0.94 & $(0.75,1.18)$ \\
\hline Intervention clinics & 943 & 0.11 & $(0.09,0.13)$ & 1163 & 0.12 & $(0.10,0.15)$ & 1.12 & $(0.85,1.46)$ \\
\hline OR & & 0.74 & $(0.57,0.97)$ & & 0.88 & $(0.70,1.12)$ & 1.19 & $(0.83,1.70)$ \\
\hline$P$ value & & & & & & & & 0.338 \\
\hline \multicolumn{9}{|l|}{ Behavioral health visits } \\
\hline Control clinics & 1061 & 0.00 & $(0.00,0.01)$ & 1473 & 0.00 & $(0.00,0.00)$ & 0.76 & $(0.18,3.15)$ \\
\hline Intervention clinics & 943 & 0.00 & $(0.00,0.01)$ & 1163 & 0.00 & $(0.00,0.00)$ & 0.45 & $(0.07,2.77)$ \\
\hline OR & & 1.13 & $(0.21,6.08)$ & & 0.67 & $(0.10,4.25)$ & 0.59 & $(0.06,5.96)$ \\
\hline$P$ value & & & & & & & & 0.655 \\
\hline \multicolumn{9}{|l|}{ Spine surgeon visits } \\
\hline Control clinics & 1061 & 0.02 & $(0.01,0.03)$ & 1473 & 0.03 & $(0.02,0.03)$ & 1.14 & $(0.69,1.90)$ \\
\hline Intervention clinics & 943 & 0.02 & $(0.01,0.03)$ & 1163 & 0.03 & $(0.02,0.04)$ & 1.27 & $(0.75,2.17)$ \\
\hline OR & & 1.02 & $(0.58,1.81)$ & & 1.14 & $(0.72,1.80)$ & 1.11 & $(0.53,2.32)$ \\
\hline$P$ value & & & & & & & & 0.777 \\
\hline \multicolumn{9}{|c|}{ Injections of lumbar spine } \\
\hline Control clinics & 1061 & 0.01 & $(0.00,0.02)$ & 1473 & 0.01 & $(0.00,0.01)$ & 0.68 & $(0.32,1.47)$ \\
\hline Intervention clinics & 943 & 0.01 & $(0.00,0.01)$ & 1163 & 0.01 & $(0.00,0.01)$ & 0.75 & $(0.28,2.00)$ \\
\hline OR & & 0.67 & $(0.26,1.68)$ & & 0.73 & $(0.28,1.92)$ & 1.10 & $(0.32,3.82)$ \\
\hline$P$ value & & & & & & & & 0.878 \\
\hline \multicolumn{9}{|c|}{ Back-related hospitalizations } \\
\hline Control clinics & 1061 & 0.01 & $(0.00,0.02)$ & 1473 & 0.02 & $(0.01,0.02)$ & 1.60 & $(0.78,3.29)$ \\
\hline Intervention clinics & 943 & 0.02 & $(0.01,0.03)$ & 1163 & 0.01 & $(0.01,0.02)$ & 0.71 & $(0.35,1.43)$ \\
\hline OR & & 1.80 & $(0.83,3.89)$ & & 0.80 & $(0.41,1.54)$ & 0.44 & $(0.16,1.21)$ \\
\hline$P$ value & & & & & & & & 0.112 \\
\hline
\end{tabular}

*Lumbar imaging includes plain films, CT, and MRI

our matched treatment options were more numerous, less familiar to clinicians, and more difficult to access, thereby placing a greater burden on our PCPs. We also used a different recruitment strategy than the English studies. Differences between the study populated in England ${ }^{9}$ and our study population could also explain outcome differences. For example, although the patient populations were similar in age, gender, employment, risk subgroup proportions, and pain severity, US patients had substantially higher baseline levels of LBP-related physical disability (RMDQ scores of 11.8 versus 8.4 , respectively).

We designed our intervention ${ }^{20}$ to be as potent as possible without making it impossible to implement in primary care clinics. Even if our intervention had improved outcomes, it may not have been feasible to implement in most US primary care settings. The high levels of burnout among PCPs and the continued turmoil in USA healthcare, ${ }^{28}$ make complex changes in clinical practice difficult.

Major strengths of the MATCH trial include randomization of matched pairs of clinics to serve as intervention or control clinics, adequate sample sizes and power to detect meaningful differences, high follow-up rates, and an adaptive and pragmatic intervention design including substantial PCP and physical therapist training, training modules based on requests of primary care teams, and inclusion of the whole primary care 
Table 5 Pre- versus post-intervention odd ratios for selected health services for LBP between the control and intervention groups during the 6 months after an index visit overall and by risk subgroup

\begin{tabular}{|c|c|c|c|c|c|c|c|c|}
\hline & \multicolumn{8}{|c|}{ All study enrollees } \\
\hline & \multicolumn{3}{|c|}{ Pre-intervention period } & \multicolumn{3}{|c|}{ Post-intervention period } & \multirow[t]{2}{*}{ OR } & \multirow[t]{2}{*}{$95 \% \mathrm{CI}$} \\
\hline & $N$ & Prop & $95 \% \mathrm{CI}$ & $N$ & Prop & $95 \% \mathrm{CI}$ & & \\
\hline \multicolumn{9}{|l|}{ Any PT visits } \\
\hline \multicolumn{9}{|l|}{ Low risk } \\
\hline Control & 134 & 0.28 & $(0.15,0.40)$ & 258 & 0.26 & $(0.16,0.36)$ & 0.93 & $(0.58,1.48)$ \\
\hline Intervention & 118 & 0.27 & $(0.14,0.40)$ & 187 & 0.31 & $(0.19,0.44)$ & 1.25 & $(0.75,2.08)$ \\
\hline $\begin{array}{l}\mathrm{OR} \\
P \text { value }\end{array}$ & & 0.97 & $(0.50,1.86)$ & & 1.30 & $(0.76,2.22)$ & 1.34 & $\begin{array}{c}(0.67,2.68) \\
0.4\end{array}$ \\
\hline \multicolumn{9}{|l|}{ Medium risk } \\
\hline Control & 120 & 0.25 & $(0.09,0.41)$ & 227 & 0.32 & $(0.15,0.50)$ & 1.45 & $(0.88,2.39)$ \\
\hline Intervention & 99 & 0.32 & $(0.11,0.53)$ & 187 & 0.34 & $(0.15,0.53)$ & 1.08 & $(0.64,1.83)$ \\
\hline OR & & 1.42 & $(0.58,3.49)$ & & 1.06 & $(0.48,2.31)$ & 0.74 & $(0.36,1.53)$ \\
\hline$P$ value & & & & & & & & 0.42 \\
\hline \multicolumn{9}{|l|}{ High risk } \\
\hline Control & 76 & 0.27 & $(0.13,0.41)$ & 129 & 0.26 & $(0.16,0.36)$ & 0.95 & $(0.50,1.82)$ \\
\hline Intervention & 56 & 0.32 & $(0.14,0.49)$ & 108 & 0.36 & $(0.22,0.50)$ & 1.20 & $(0.61,2.40)$ \\
\hline OR & & 1.24 & $(0.58,2.67)$ & & 1.57 & $(0.90,2.73)$ & 1.26 & $(0.49,3.25)$ \\
\hline$P$ value & & & & & & & & 0.63 \\
\hline \multirow{2}{*}{\multicolumn{9}{|c|}{$\begin{array}{l}\text { Any CAM visits } \\
\text { Low risk }\end{array}$}} \\
\hline & & & & & & & & \\
\hline Control & 134 & 0.17 & $(0.08,0.26)$ & 258 & 0.13 & $(0.08,0.18)$ & 0.71 & $(0.39,1.31)$ \\
\hline Intervention & 118 & 0.11 & $(0.04,0.17)$ & 187 & 0.14 & $(0.08,0.20)$ & 1.38 & $(0.67,2.84)$ \\
\hline OR & & 0.57 & $(0.27,1.23)$ & & 1.11 & $(0.62,2.01)$ & 1.94 & $(0.75,5.01)$ \\
\hline$P$ value & & & & & & & & 0.17 \\
\hline \multicolumn{9}{|l|}{ Medium risk } \\
\hline Control & 120 & 0.19 & $(0.10,0.28)$ & 227 & 0.17 & $(0.11,0.23)$ & 0.85 & $(0.47,1.55)$ \\
\hline Intervention & 99 & 0.13 & $(0.05,0.21)$ & 187 & 0.11 & $(0.06,0.16)$ & 0.82 & $(0.39,1.76)$ \\
\hline OR & & 0.64 & $(0.30,1.37)$ & & 0.61 & $(0.34,1.09)$ & 0.96 & $(0.37,2.52)$ \\
\hline$P$ value & & & & & & & & 0.94 \\
\hline \multicolumn{9}{|l|}{ High risk } \\
\hline Control & 76 & 0.27 & $(0.13,0.41)$ & 129 & 0.26 & $(0.16,0.36)$ & 0.95 & $(0.50,1.82)$ \\
\hline Intervention & 56 & 0.32 & $(0.14,0.49)$ & 108 & 0.36 & $(0.22,0.50)$ & 1.20 & $(0.61,2.40)$ \\
\hline OR & & 1.24 & $(0.58,2.67)$ & & 1.57 & $(0.90,2.73)$ & 1.26 & $(0.49,3.25)$ \\
\hline$P$ value & & & & & & & & 0.63 \\
\hline Any LBP ima & & & & & & & & \\
\hline Low risk & & & & & & & & \\
\hline Control & 134 & 0.16 & $(0.00,0.32)$ & 258 & 0.20 & $(0.02,0.39)$ & 1.30 & $(0.70,2.44)$ \\
\hline Intervention & 118 & 0.25 & $(0.01,0.49)$ & 187 & 0.18 & $(0.01,0.35)$ & 0.65 & $(0.37,1.15)$ \\
\hline OR & & 1.74 & $(0.44,6.91)$ & & 0.86 & $(0.23,3.20)$ & 0.50 & $(0.21,1.16)$ \\
\hline$P$ value & & & & & & & & 0.11 \\
\hline Medium risk & & & & & & & & \\
\hline Control & 120 & 0.25 & $(0.07,0.44)$ & 227 & 0.16 & $(0.05,0.28)$ & 0.57 & $(0.33,1.00)$ \\
\hline Intervention & 99 & 0.25 & $(0.06,0.44)$ & 187 & 0.28 & $(0.09,0.47)$ & 1.19 & $(0.68,2.08)$ \\
\hline OR & & 0.98 & $(0.34,2.83)$ & & 2.03 & $(0.76,5.40)$ & 2.07 & $(0.94,4.57)$ \\
\hline$P$ value & & & & & & & & 0.07 \\
\hline High risk & & & & & & & & \\
\hline Control & 76 & 0.34 & $(0.17,0.52)$ & 129 & 0.34 & $(0.20,0.47)$ & 0.96 & $(0.53,1.77)$ \\
\hline Intervention & 56 & 0.29 & $(0.11,0.47)$ & 108 & 0.30 & $(0.17,0.44)$ & 1.06 & $(0.52,2.17)$ \\
\hline OR & & 0.79 & $(0.36,1.76)$ & & 0.87 & $(0.47,1.59)$ & 1.10 & $(0.43,2.80)$ \\
\hline$P$ value & & & & & & & & 0.85 \\
\hline Any narcotic & & & & & & & & \\
\hline Low risk & & & & & & & & \\
\hline Control & 134 & 0.28 & $(0.16,0.41)$ & 258 & 0.23 & $(0.16,0.31)$ & 0.76 & $(0.46,1.27)$ \\
\hline Intervention & 118 & 0.34 & $(0.19,0.48)$ & 187 & 0.24 & $(0.15,0.33)$ & 0.62 & $(0.36,1.08)$ \\
\hline OR & & 1.29 & $(0.71,2.33)$ & & 1.06 & $(0.65,1.73)$ & 0.82 & $(0.39,1.73)$ \\
\hline$P$ value & & & & & & & & 0.61 \\
\hline Medium risk & & & & & & & & \\
\hline Control & 120 & 0.56 & $(0.34,0.78)$ & 227 & 0.47 & $(0.34,0.61)$ & 0.70 & $(0.43,1.13)$ \\
\hline Intervention & 99 & 0.48 & $(0.27,0.68)$ & 187 & 0.48 & $(0.33,0.63)$ & 1.00 & $(0.59,1.70)$ \\
\hline OR & & 0.71 & $(0.40,1.27)$ & & 1.02 & $(0.67,1.56)$ & 1.43 & $(0.70,2.92)$ \\
\hline$P$ value & & & & & & & & 0.33 \\
\hline High risk & & & & & & & & \\
\hline Control & 76 & 0.72 & $(0.33,1.11)$ & 129 & 0.67 & $(0.39,0.95)$ & 0.81 & $(0.42,1.55)$ \\
\hline Intervention & 56 & 0.66 & $(0.26,1.05)$ & 108 & 0.61 & $(0.33,0.88)$ & 0.81 & $(0.40,1.64)$ \\
\hline & & 0.74 & $(0.33,1.68)$ & & 0.74 & $(0.40,1.39)$ & 1.00 & $(0.38,2.61)$ \\
\hline$P$ value & & & & & & & & 1.00 \\
\hline
\end{tabular}

Entire study cohort $(n=1699)$ 
team. ${ }^{20}$ Limitations include less than half of all LBP patients participated and may not be representative of all patients, the need to defer baseline data collection until 2 weeks after the PCP visit thereby missing any early treatment effects, and the restriction to a single socio-economically homogenous integrated healthcare system.

\section{CONCLUSIONS}

In contrast to the positive results of implementing a risk stratification strategy to improve primary care for LBP in England, ${ }^{15,16}$ our adaptation of that strategy to the different circumstances in our setting did not change healthcare utilization or improve patient outcomes. This illustrates the risk of failure when complex interventions developed and found effective in one setting are implemented in a different setting even with strong system support and substantial resources devoted to adapting the intervention to local needs and circumstances.

To increase their chances of success, future initiatives to implement complex interventions in primary care should include simple and easily implemented and supported treatment recommendations, automatic alerts in the EHR to make it easy for clinicians to remember to collect riskstratification information and recommend appropriate matched treatments to their patients, and the provision of regular feedback on their performance adhering to the matched treatment recommendations for patients at each risk stratum are likely to improve the chances of success. Given the limited ability of primary care clinicians to take on new responsibilities, however, innovative approaches (e.g., expanded nurse role) may be necessary to promote the clinical changes necessary to improve patient outcomes.

Corresponding Author: Dan Cherkin, PhD; Kaiser Permanente Washington Health Research Institute, 1730 Minor Avenue, Suite 1600, Seattle, WA 98101, USA (e-mail: cherkin.d@ghc.org).

Contributors We are very grateful to the primary care clinicians, physical therapists, and staff at the Group Health Lynnwood, Northgate, and Renton clinics for their support for and engagement in the intervention. In addition, we would like to thank the following members of the research team; Zoe Bermet, Kristin Delaney, MPH, John Ewing, Kevin Filocamo, Vina Graham, and Margie Wilcox, and Survey Interviewers; Jennifer Brandlin, Donna Luce, and Patricia Scott.

Funding Support Funding for this trial was provided by the Patient Centered Care Research Institute ("Evaluation of a Patient-Centered Risk Stratification Method for Improving Primary Care for Back Pain": Contract \#398) and by the National Center for Complementary and Integrative Health/NIH ("Implementing Evidence-Based Treatments for Persistent Back Pain into Primary Care": Grant No. R21AT0007326). Martin Levine, Diane Piekara, and Pam Rock received support to participate in the quality improvement activities from Group Health. Nadine E Foster, an NIHR Senior Investigator, and Jonathan C. Hill were supported through an NIHR Research Professorship (NIHR-RP011-015) awarded to Nadine Foster. The views expressed are those of the authors and not necessarily those of the Patient Centered Care Research Institute, NIH, NHS, the NIHR, or the Department of Health.

\section{Compliance with Ethical Standards:}

Conflict of Interest: The authors declare that they do not have a conflict of interest.

Authors' Information: Our research team included four "patient partners" (GB, $M J, A L, J Y)$ with chronic back pain who provided their unique and valuable expert advice on the trial materials and processes.

Disclaimer: The views and opinions expressed in this paper are those of the authors and do not necessarily reflect those of the PatientCentered Outcomes Research Institute, the National Institutes of Health, NIHR, NHS, or the Department of Health. None of the agencies that funded the trial had any input into the trial design, conduct, data collection, analysis or interpretation, reporting, or writing of this article.

\section{REFERENCES}

1. Martin BI, Deyo RA, Mirza SK, Turner JA, Comstock BA, Hollingworth W, Sullivan SD. Expenditures and health status among adults with back and neck problems. JAMA 2008;299:656-64.

2. Von Korff M, Kolodny A, Deyo RA, Chou R. Long-term opioid therapy reconsidered. Ann Intern Med 2011;155:325-8.

3. Von Korff M, Franklin G. Responding to America's iatrogenic epidemic of prescription opioid addiction and overdose. Wolters Kluwer Health, Inc.; Philadelphia 2016.

4. Moore JE. Chronic low back pain and psychosocial issues. Phys Med Rehabil Clin N Am 2010;21(4):801-15.

5. Boothby JL, Thorn BE, Stroud MW, Jensen MP. Coping with pain. In: Gatchel RJ, Turk DC, editors. Psychosocial factors in pain: critical perspectives. New York: Guilford Press; 1999: 343-59.

6. Jensen MP, Karoly P. Control beliefs, coping efforts, and adjustment to chronic pain. J Consult Clin Psychol 1991;59:431-8.

7. Hill JC, Dunn KM, Lewis M, Mullis R, Main CJ, Foster NE, Hay EM. A primary care back pain screening tool: identifying patient subgroups for initial treatment. Arthritis Rheum 2008;59:632-41.

8. Hill JC, Whitehurst DG, Lewis M, et al. Comparison of stratified primary care management for low back pain with current best practice (STarT Back): a randomised controlled trial. Lancet 2011;378:1560-71.

9. Foster NE, Mullis R, Hill JC, et al. Effect of stratified care for low back pain in family practice (IMPaCT Back): a prospective population-based sequential comparison. Ann Fam Med 2014;12:102-11.

10. Whitehurst DG, Bryan S, Lewis M, Hay EM, Mullis R, Foster NE. Implementing stratified primary care management for low back pain: cost-utility analysis alongside a prospective, population-based, sequential comparison study. Spine 2015;40(4):405-414..

11. Hay EM, Dunn KM, Hill JC, et al. A randomised clinical trial of subgrouping and targeted treatment for low back pain compared with best current care. The STarT Back Trial Study Protocol BMC Musculoskelet Disord 2008;9:58.

12. Hill JC, Vohora K, Dunn KM, Main CJ, Hay EM. Comparing the STarT back screening tool's subgroup allocation of individual patients with that of independent clinical experts. Clin J Pain 2010;26:783-7.

13. Sowden $\mathrm{G}$, Hill J, Konstantinou $\mathrm{K}$, et al. Subgrouping for targeted treatment in primary care for low back pain: the treatment system and clinical training programmes used in the IMPaCT Back study (ISRCTN 55174281). Fam Pract 2012; 29(1):50-62.

14. Main CJ, Sowden G, Hill JC, Watson PJ, Hay EM. Integrating physical and psychological approaches to treatment in low back pain: the development and content of the STarT Back trial's 'high-risk' intervention (StarT Back; ISRCTN 37113406) Physiotherapy 2012; 98; 2: 110-117.

15. Murphy SE, Blake C, Power CK, Fullen BM. Comparison of a stratified group intervention (STarT Back) with usual group care in patients with low back pain: a non-randomised controlled trial. Spine (Phila Pa 1976). 2016; 41(8):645-52.

16. Karlen E, McCathie B. Implementation of a quality improvement process aimed to deliver higher-value physical therapy for patients with low back pain: case report. Phys Ther 2015;95(12):1712-21.

17. Karstens S, Krug K, Hill JC, Stock C, Steinhaeuser J, Szecsenyi J, et al. Validation of the German version of the STarT-Back Tool (STarT-G): a cohort study with patients from primary care practices. BMC Musculoskelet Disord 2015;16:346. 
18. Karstens S, Joos S, Hill JC, Krug K, Szecsenyi J, Steinhauser J. General practitioners views of implementing a stratified treatment approach for low back pain in Germany: a qualitative study. PLoS One 2015;10(8):e0136119.

19. Matsudaira K, Oka H, Kikuchi N, Haga Y, Sawada T, Tanaka S. Psychometric properties of the Japanese version of the STarT back tool in patients with low back pain. PLoS One 2016;11(3):e0152019.

20. Cherkin D, Balderson B, Brewer G et al. Evaluation of a risk-stratification strategy to improve primary care for back pain: the MATCH cluster randomized trial protocol. BMC Musculoskelet Disord 2016;17:361.

21. Hemming $\mathrm{K}$, Haines TP, Chilton $\mathrm{AJ}$, et al. The stepped wedge cluster randomized trial: rationale, design, analysis, and reporting. BMJ 2015;351:h391.

22. Bombardier C, Hayden J, Beaton DE. Minimal clinically important difference. Low back pain: outcome measures. J Rheumatol 2001;28(2):431-8.

23. Chiarotto A, Deyo RA, Terwee CB, et al. Core outcome domains for clinical trials in non-specific low back pain. Eur Spine J 2015;24(6):1127-42.

24. Laird NM, Ware JH. Random-effects models for longitudinal data. Biometrics 1982;38:963-74

25. Pinheiro JC, Bates D.M. Approximations to the log-likelihood function in the nonlinear mixed-effects model. J Comput Graph Stat; 1995;4:12-35.

26. Suri P, Delaney K, Rundell SD, Cherkin DC. Predictive validity of the STarT back tool for risk of persistent disabling back pain in a U.S primary care setting. Arch Phys Med Rehabil. 2018;S0003-9993(18)30204-1. https://doi.org/10.1016/j.apmr.2018.02.016

27. Riis A, Jensen CE, Bro F, Maindal HT, Petersen KD, Bendtsen MD, Jensen MB. A multifaceted implementation strategy versus passive implementation of low back pain guidelines in general practice: a cluster randomised controlled trial. Implement Sci 2016;11(1):143.

28. Shanafelt TD, Dyrbye LN, West CP. Addressing physician burnout: the way forward. JAMA. 2017; 317(9): 901-902.

\section{APPENDIX 1: THE CARE IMPROVEMENT INTERVENTION}

(See ref. ${ }^{20}$ for more details)

The care improvement intervention included several interrelated and synergistic components.

\section{Incorporating Decision Support Tools into Electronic} Health Record. After clinicians or nursing staff entered patients' responses to the STarT Back questions, the electronic health record (EHR) automatically calculated each patient's risk stratum and displayed recommended treatment options for that stratum on a screen visible to clinicians and patients. The goal was to provide an opportunity for clinicians to discuss treatment options with their patients. Because nursing staff sometimes collected and entered patient responses to the STarT Back Tool, provider use of the tool varied greatly, and, therefore, it is unclear how many providers used the tools as intended with patients. In addition, shortcuts were incorporated into the EHR to help clinicians efficiently access the STarT Back Tool, the Group Health $(\mathrm{GH})$ back pain guidelines, existing GH educational resources (DVDs about acute and chronic back pain, and when surgery might be indicated), and GH's self-management groups for persons with chronic conditions (Living Well with Chronic Conditions).

\section{Identifying Recommended Treatment Options for Patients in} Each Risk Stratum. We used the STarT Back tool without modification. However, we relied on GH's new low back pain guidelines to identify several evidence-based treatment options available at GH appropriate for each patient subgroup. This resulted in the following treatment recommendations for each risk stratum:
- Low risk ( $\sim 40 \%$ of patients): Conduct a brief assessment to rule out potentially serious causes of back pain (i.e., "red flags"), do not refer to other healthcare professionals, elicit and listen to patients' concerns, provide reassurance about the positive prognosis and self-care recommendations to relieve pain (physical activity, pain medications, avoiding bed rest). Encourage patients to access online DVDs that reinforced information about acute or chronic back pain and the importance of self-care.

- Moderate risk ( $\sim 40 \%$ of patients): In addition to ruling out red flags and encouraging self-care, recommend activating treatments such as physical therapist-led exercise and yoga that could reduce fear of movement. For patients not interested in activating treatments consider more passive options (acupuncture, chiropractic, or massage therapy) in the hope these treatments will help decrease their pain and prepare them for more active approaches.

- High risk ( $\sim 20 \%$ of patients): In addition to ruling out red flags, recommend or refer patients to the GH PTs who were specially trained in the MATCH trial to offer a systematic approach to the integration of physical and psychological approaches to treatment of people with low back pain. Another evidence-based approach for chronic back pain, cognitive behavioral therapy (CBT) from a psychologist, could be recommended, but access was very limited. PCPs were also encouraged to proactively follow-up with high-risk patients within 2 weeks.

Training for Primary Care Teams. Six one-hour training sessions on separate topics were given in each of the three primary care intervention clinics. Each topic was presented on several occasions to ensure that all primary care providers (PCPs) participated. Sessions were presented roughly monthly over a 6-month period (May-October 2014). Training focused on the STarT Back tool and matched treatment options (emphasizing the importance of the biopsychosocial model), techniques and strategies for talking about chronic pain with patients, the special training GH physical therapists (PTs) had received in incorporating simple CBT techniques into their PT practice to use with high-risk patients, and understanding the role of evidence-based complementary and alternative medial (CAM) therapies. Education about CAM providers was particularly important because, despite a Washington State law mandating access to these evidence-based treatments, few PCPs know much about them and rarely mention them as treatment options to their patients. GH contracted with an external network of CAM providers to provide these services.

Physical therapists and members of the nursing staff were invited to attend several of the sessions. In addition, PCPs and staff received coaching on how to locate and correctly use the STarT Back and other related tools in the EHR. Most PCPs participated in at least one such coaching session. Finally, to reduce knowledge barriers to recommending matched treatment options, we compiled a list of the names and contact 
information of recommended local CAM providers and made them available to clinic staff.

Training for Physical Therapists. The PTs in the intervention clinics received 5 days of training from an instructor from England (GS) who had trained the PTs in the original studies of the STarT Back strategy. This training aimed to provide PTs with a better understanding of how psychosocial factors contribute to the pain experience and helped them apply painrelevant psychosocial theories and practice to maximize their effectiveness in reducing their patients' pain-related disability.

\section{APPENDIX 2}

Table 6 Patient outcomes at 2-month follow-up

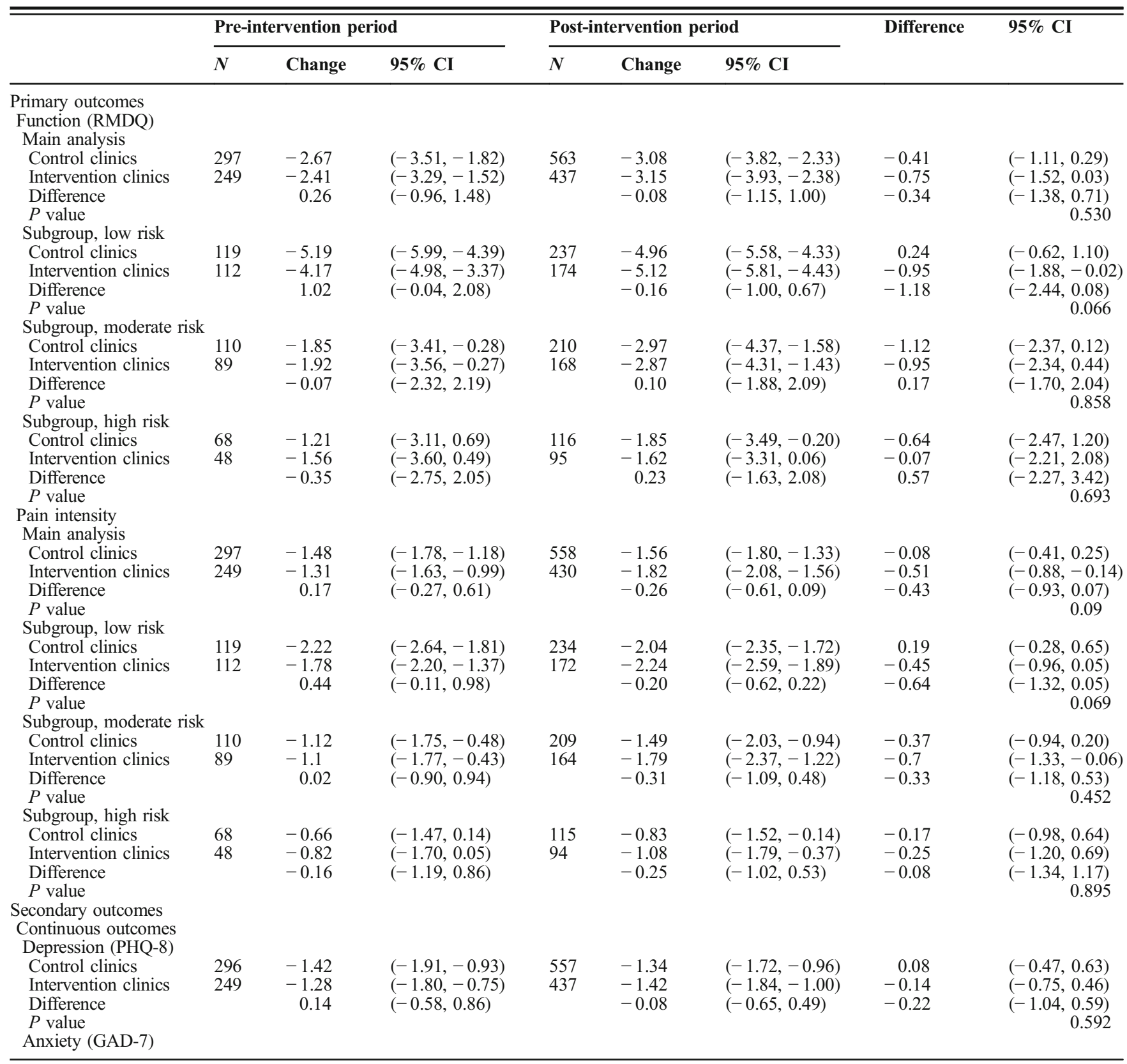


Table 6. (continued)

\begin{tabular}{|c|c|c|c|c|c|c|c|c|}
\hline & \multicolumn{3}{|c|}{ Pre-intervention period } & \multicolumn{3}{|c|}{ Post-intervention period } & \multirow[t]{2}{*}{ Difference } & \multirow[t]{2}{*}{$95 \% \mathrm{CI}$} \\
\hline & $N$ & Change & $95 \% \mathrm{CI}$ & $N$ & Change & $95 \% \mathrm{CI}$ & & \\
\hline Control clinics & 297 & 0.76 & $(0.38,1.14)$ & 557 & 0.88 & $(0.60,1.16)$ & 0.12 & $(-0.36,0.60)$ \\
\hline Intervention clinics & 249 & 0.87 & $(0.45,1.29)$ & 436 & 0.71 & $(0.39,1.03)$ & -0.16 & $(-0.68,0.37)$ \\
\hline Difference & & 0.11 & $(-0.46,0.68)$ & & -0.17 & $(-0.59,0.26)$ & -0.28 & $(-0.99,0.44)$ \\
\hline$P$ value & & & & & & & & 0.448 \\
\hline \multicolumn{9}{|c|}{ Fear of movement (TSK) } \\
\hline Control clinics & 297 & -2.92 & $(-3.92,-1.91)$ & 555 & -2.8 & $(-3.59,-2.02)$ & 0.11 & $(-1.02,1.25)$ \\
\hline Intervention clinics & 248 & -2.51 & $(-3.59,-1.42)$ & 434 & -3.33 & $(-4.19,-2.47)$ & -0.82 & $(-2.09,0.44)$ \\
\hline Difference & & 0.41 & $(-1.07,1.89)$ & & -0.53 & $(-1.70,0.64)$ & -0.94 & $(-2.64,0.76)$ \\
\hline$P$ value & & & & & & & & 0.28 \\
\hline \multicolumn{9}{|l|}{ Self-efficacy (PSEQ) } \\
\hline Control clinics & 296 & 2.53 & $(1.30,3.76)$ & 561 & 2.87 & $(1.89,3.84)$ & 0.34 & $(-1.00,1.68)$ \\
\hline Intervention clinics & 249 & 2.55 & $(1.23,3.87)$ & 434 & 2.87 & $(1.80,3.94)$ & 0.32 & $(-1.17,1.80)$ \\
\hline Difference & & 0.02 & $(-1.78,1.83)$ & & 0 & $(-1.45,1.45)$ & -0.02 & $(-2.03,1.98)$ \\
\hline$P$ value & & & & & & & & 0.982 \\
\hline \multicolumn{9}{|c|}{ Effect on work productivity $(0-10)$} \\
\hline Control clinics & 161 & -1.06 & $(-1.37,-0.74)$ & 303 & -1.19 & $(-1.42,-0.95)$ & -0.13 & $(-0.50,0.24)$ \\
\hline Intervention clinics & 123 & -1.23 & $(-1.58,-0.87)$ & 226 & -1.16 & $(-1.43,-0.89)$ & 0.07 & $(-0.36,0.49)$ \\
\hline Difference & & -0.17 & $(-0.64,0.31)$ & & 0.03 & $(-0.33,0.39)$ & 0.19 & $(-0.37,0.76)$ \\
\hline$P$ value & & & & & & & & 0.499 \\
\hline \multicolumn{9}{|c|}{ Hours of work lost due to back pain } \\
\hline Control clinics & 162 & -2.15 & $(-2.93,-1.37)$ & 299 & -2.65 & $(-3.23,-2.08)$ & -0.5 & $(-1.47,0.46)$ \\
\hline Intervention clinics & 123 & -3.4 & $(-4.29,-2.50)$ & 227 & -2.69 & $(-3.35,-2.03)$ & 0.71 & $(-0.40,1.83)$ \\
\hline Difference & & -1.25 & $(-2.43,-0.06)$ & & -0.03 & $(-0.91,0.85)$ & 1.22 & $(-0.26,2.69)$ \\
\hline$P$ value & & & & & & & & 0.107 \\
\hline \multicolumn{9}{|l|}{ Binary outcomes } \\
\hline \multicolumn{9}{|c|}{ Completely recovered or much better (PGIC) } \\
\hline Control clinics & 296 & 0.27 & $(0.16,0.38)$ & 554 & 0.3 & $(0.21,0.38)$ & 1.14 & $(0.70,1.84)$ \\
\hline Intervention clinics & 249 & 0.21 & $(0.12,0.31)$ & 432 & 0.33 & $(0.22,0.44)$ & 1.85 & $(1.07,3.19)$ \\
\hline OR & & 0.72 & $(0.40,1.30)$ & & 1.18 & $(0.76,1.81)$ & 1.63 & $(0.79,3.37)$ \\
\hline$P$ value & & & & & & & & 0.189 \\
\hline \multicolumn{9}{|c|}{ Very satisfied with care } \\
\hline Control clinics & 285 & 0.47 & $(0.31,0.63)$ & 531 & 0.54 & $(0.41,0.68)$ & 1.35 & $(0.88,2.05)$ \\
\hline Intervention clinics & 240 & 0.36 & $(0.22,0.50)$ & 425 & 0.48 & $(0.34,0.61)$ & 1.61 & $(1.00,2.58)$ \\
\hline OR & & 0.65 & $(0.39,1.07)$ & & 0.77 & $(0.53,1.12)$ & 1.19 & $(0.63,2.24)$ \\
\hline \multirow{2}{*}{\multicolumn{9}{|c|}{ Very satisfied with treatment }} \\
\hline & & & & & & & & \\
\hline Control clinics & 243 & 0.35 & $(0.22,0.48)$ & 434 & 0.3 & $(0.21,0.39)$ & 0.81 & $(0.50,1.29)$ \\
\hline Intervention clinics & 205 & 0.28 & $(0.16,0.40)$ & 344 & 0.31 & $(0.21,0.41)$ & 1.13 & $(0.67,1.91)$ \\
\hline OR & & 0.73 & $(0.42,1.28)$ & & 1.03 & $(0.67,1.58)$ & 1.4 & $(0.69,2.84)$ \\
\hline & & & & & & & & 0.344 \\
\hline \multicolumn{9}{|c|}{ Very satisfied with information about cause } \\
\hline Control clinics & 288 & 0.31 & $(0.19,0.43)$ & 530 & 0.38 & $(0.27,0.49)$ & 1.38 & $(0.85,2.23)$ \\
\hline Intervention clinics & 247 & 0.29 & $(0.16,0.41)$ & 427 & 0.34 & $(0.23,0.45)$ & 1.3 & $(0.76,2.20)$ \\
\hline OR & & 0.894 & $0.5,1.59$ & & 0.84 & $0.55,1.29$ & 0.94 & $0.46,1.92$ \\
\hline$P$ value & & & & & & & & 0.864 \\
\hline
\end{tabular}

